\title{
Effects of Viscous Dissipation and Joule Heating on Micropolar Hybrid Nanofluid in a Stretching/Shrinking Channel including Thermal/Solar Radiation
}

\author{
H.A El-dawy ( $\sim$ ha_sa3000@yahoo.com ) \\ High Institute of Engineering and Technology Tod, Luxor, Egypt \\ Mohamed El-Amin \\ College of Engineering, Effat University, Jeddah, KSA
}

\section{Research Article}

Keywords: hybrid nanofluid ,micropolar , heat transfer , radiation, Joule Heating

Posted Date: July 15th, 2021

DOl: https://doi.org/10.21203/rs.3.rs-710400/v1

License: (c) (i) This work is licensed under a Creative Commons Attribution 4.0 International License.

Read Full License 


\title{
Effects of Viscous Dissipation and Joule Heating on Micropolar Hybrid Nanofluid in a Stretching/Shrinking Channel including Thermal/Solar Radiation
}

\author{
H. A. El-dawy ${ }^{1, *}$ and Mohamed F. El-Amin ${ }^{2,3}$ \\ ${ }^{1}$ High Institute of Engineering and Technology Tod, Luxor, Egypt \\ ${ }^{2}$ College of Engineering, Effat University, Jeddah, KSA \\ ${ }^{3}$ Department of Mathematics, Faculty of Science, Aswan University, Aswan, Egypt \\ Emails: ha_sa3000@yahoo.com; mfam2000@yaho.com
}

\begin{abstract}
:
This study aims to investigate the behavior of the micropolar hybrid nanofluid $\left(\mathrm{Cu}-\mathrm{TiO}_{2}\right)$ in a stretching and shrinking channel while maintaining a continuous laminar flow. External effects from magnetic field, thermal and solar radiation are also considered in the model. The governing differential (momentum, micro-rotation, and energy) equations are transformed into dimensionless forms via similarity transformations based on the boundary layer theory. The resulting ordinary differential equations are solved numerically using the fourth-order Runge-Kutta-Gill technique with the shooting method. The effects of nanoparticles transport on fluid flow and heat transfer are discussed and compared to the pure water case. The results are presented in tables or graphs such as velocity, temperature, angular-velocity profiles, and concentration.
\end{abstract}

Keyword: hybrid nanofluid -micropolar - heat transfer - radiation- Joule Heating.

\section{Introduction}

On the other hand, micropolar fluids have a microstructure, including fluids having nonsymmetrical stress sensors. They are essential in hydrodynamic-fluid problems because they are made up of randomly oriented particles suspended in a viscous medium. Micropolar fluids have a wide range of applications, such as extrusion of plastic sheets, paper production, and glass blowing [3,4,5]. Eringen [3] was the first to formulate the theory of micro-polar fluids. Srinivasacharya et al. [6] reviewed the entropy production and heat transfer of micro-polar fluid flow in an annulus with a magnetic field. A computational study into the heat and mass transfer of a micro-polar fluid flow over a stretching surface was conducted by Mabood et al. [7]. Many other researchers have studied micropolar fluid flow researchers [8-13]. Ellahi et al. [14] investigated mixed convection nanofluid flow over a wedge in a porous medium. 
Akbarzadeh et al. [15] have studied laminar nanofluid flow and force convective heat transfer within a wavy tube. Ellahi et al. [16] produced a two-dimensional heat transfer flow of a nanofluid in the vicinity of a stagnation stage. Sheikholeslami and Ganji [17] studied the hydrothermal activity of nanofluid flow between two parallel plates in the presence of a magnetic field. The magneto-hydrodynamic nanofluid flow between parallel sheets was studied by Sheikholeslami and Ganji [18]. Rahbari et al. [19] conducted an analytical analysis of blood flow, including nanoparticles, through porous vessels. EndoScopic study of peristaltic blood flow of Sisko fluid with Titanium magneto-nanoparticles through a uniform tube was performed by Bhatti et al. [20]. Bhatti et al. [21, 22] looked at the effects of coagulation (blood clot) and a variable magnetic field on three-dimensional unsteady MHD boundary layer flow of viscous nanofluid containing gyrotactic microorganisms. The combined impact of thermodiffusion and thermal radiation on Williamson fluid was studied by Bhatti and Rashidi [23]. Rashidi et al. [24] investigated heat transfer over a stretching sheet in a nanofluid flow. Sheremet et al. [25] investigated the unsteady natural convection of a water-based nanofluid inside a wavy-walled cavity under the influence of a uniform inclined magnetic field. Free convection was studied by Sheremet et al. [26] in a square differentially heated porous cavity filled with a nanofluid. Sheremet et al. [27] investigated steady-state natural convection heat transfer between non-parallel walls in a three-dimensional MHD nanofluid flow. Flow and heat transfer between two flat plates of magneto-hydrodynamic (MHD) nanofluid by Dogonchi et al. [28]. Dogonchi and Ganji [29] investigated the Brownian motion effect of nanofluid flow and heat transfer between non-parallel stretching walls. Dogonchi et al. [30] studied the unsteady squeezing flow and heat transfer in MHD. The flow of nanofluids near a stagnation point was studied by El-dawy et al. [31]. El-dawy and Gorla [32] examined the turbulent flow of a nanofluid over a wedge. As cooling and heating fluids, nanofluids in minichannels of different configurations are used [33]. In the presence of injection and suction, heat transfer and fluid flow of a pseudo-plastic non-Newtonian nanofluid over a permeable surface have been overcome. Maleki et al. [34] studied nanofluid flow in microchannel heat sinks of similar length and hydraulic diameter by Goodarzi et al. [35].

The effects of hybrid nanoparticles $(\mathrm{Cu}-\mathrm{TiO} 2)$ on micropolar nanofluid flow, heat transfer and concentration in stretching and shrinking channel with radiations and heat source are studied in this paper. The paper is organized as follows. In Sec. 2, the problem is described and the mathematical modeling is presented. Then, the results are 
presented and discussed in Sec. 3. Finally, conclusion is drawn in Sec. 4.

\section{Problem description}

In a two-dimensional tube, consider the steady laminar flow of a micropolar hybrid nanofluid. The channel walls are in two cases stretching and shrinking, and the fluid enters the channel or exits by the uniform speed of $\mathrm{v}_{0}$. The temperature of bottom wall is defined by $T_{1}$, while $T_{2}$ indicates the temperature of top wall. The channel walls are located at $y= \pm h$ (see Fig. 1).

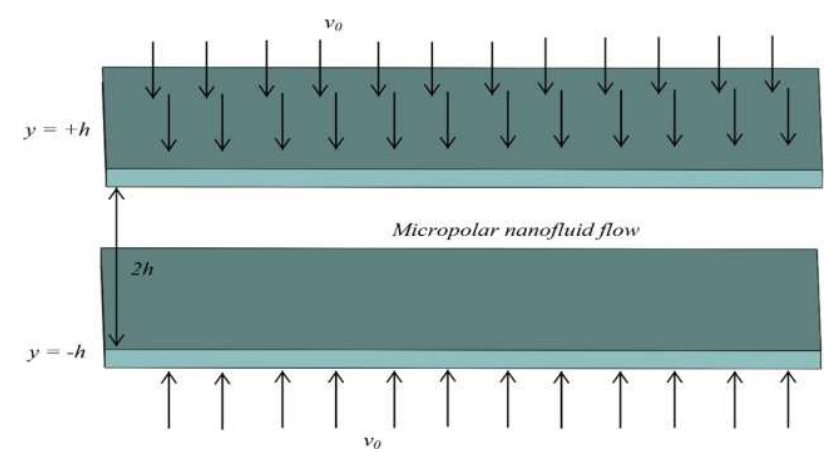

Fig. 1: Schematic diagram of the problem under consideration

It is assumed that there is a thermal equilibrium between the nanoparticles and the base fluid and there is no slip velocity between the phases. The governing boundary layer equations of momentum, energy, micro-rotation and energy are:

$\frac{\partial u}{\partial x}+\frac{\partial v}{\partial y}=0$

$\rho_{h f}\left(u \frac{\partial u}{\partial x}+v \frac{\partial u}{\partial y}\right)=a^{2} x+\left(\mu_{h f}+k\right) \frac{\partial^{2} u}{\partial y^{2}}+k \frac{\partial N}{\partial y}+\sigma_{f} B^{2}(a x-u)+g(T-$

$\left.T_{\infty}\right)\left(\rho \beta_{T}\right)_{h f}+\left(C-C_{\infty}\right)\left(\rho \beta_{C}\right)_{h f}$

$\rho_{h f} j\left(u \frac{\partial N}{\partial x}+v \frac{\partial N}{\partial y}\right)=\gamma_{h f} \frac{\partial^{2} N}{\partial y^{2}}-k\left(2 N+\frac{\partial u}{\partial y}\right)$

$u \frac{\partial T}{\partial x}+v \frac{\partial T}{\partial y}=\propto_{h f}\left(\frac{\partial^{2} T}{\partial y^{2}}\right)+\frac{1}{\left(\rho C_{p}\right)_{h f}} \frac{\partial q_{r a d}}{\partial y}+Q_{0}\left(T-T_{w}\right)+\mu_{h f}\left(\frac{\partial u}{\partial x}\right)^{2}+\frac{\sigma_{h f} B^{2}}{\left(\rho C_{p}\right)_{h f}} u^{2}$

$u \frac{\partial C}{\partial x}+v \frac{\partial C}{\partial y}=D_{h f} \frac{\partial^{2} C}{\partial y^{2}}-K_{r}\left(C-C_{w}\right)$

where $\mathrm{u}$ and $\mathrm{v}$ are the velocity components, $\mathrm{P}$ is the pressure, $\mathrm{T}$ is the temperature, $\sigma_{h f}, Q_{0}$ is the electric conductivity of the fluid, Volumetric rate of heat generation/ absorption, $\mathrm{N}$ is the microrotation velocity, $\mathrm{k}$ is the vortex viscosity, $\gamma_{h f}=\left(\mu_{h f}+\frac{K}{2}\right) j$ 
is the spin gradient viscosity, $\mathrm{j}$ is the micro-inertia density and $\mathrm{g}$ is the gravity, $\mathrm{T}, \mathrm{T}_{\mathrm{W}}$, $\mathrm{T}_{\infty}$ and the temperature of the fluid inside the thermal boundary layer, the plate and the free-stream flow, respectively; $\rho_{n f}, k_{h f}, \mu_{h f},\left(C_{p}\right)_{h f}$ are density, thermal conductivity, dynamic viscosity and specific heat of the hybrid nanofluid respectively,.$q$ rad is the radiative heat flux, $k_{\mathrm{r}}$ (reaction rate).

We consider Rosseland approximation to express the for radiation:

$q_{\text {rad }}=\frac{-4 \sigma^{*}}{3 k_{h f}^{*}} \frac{\partial T^{4}}{\partial y}$

where $\sigma^{*}$ is the Stefan-Boltzmann constant and $k_{h f}^{*}$ is the mean absorption coefficient of the nanofluid. Further, such that $T^{4}$ may be expanded in a Taylor series. So, expanding $T^{4}$ about $T_{\infty}$ and ignoring higher order terms we get,

$$
T^{4}=4 T_{\infty}^{3} T-3 T_{\infty}^{4}
$$

Table 1: some Physical Relations [33]

\begin{tabular}{|c|c|c|}
\hline Density & $\rho_{n f}=\rho_{f}\left((1-\varphi)+\varphi\left(\frac{\rho_{s}}{\rho_{f}}\right)\right)$ & $\rho_{h f}=\rho_{f}\left(1-\varphi_{2}\right)\left(\left(1-\varphi_{1}\right)+\varphi_{1}\left(\frac{\rho_{s 1}}{\rho_{f}}\right)\right)+\varphi_{2} \rho_{s 2}$ \\
\hline Viscosity & $\mu_{n f}=\frac{\mu_{f}}{(1-\varphi)^{2.5}}$ & $\mu_{h f}=\frac{\mu_{f}}{\left(1-\varphi_{1}\right)^{2.5}\left(1-\varphi_{2}\right)^{2.5}}$ \\
\hline $\begin{array}{l}\text { Heat } \\
\text { capacity }\end{array}$ & $\begin{aligned}\left(\rho C_{p}\right)_{n f}=\left(\rho C_{p}\right)_{f} & (1-\varphi \\
& \left.-\varphi \frac{\left(\rho C_{p}\right)_{s}}{\left(\rho C_{p}\right)_{f}}\right)\end{aligned}$ & $\begin{array}{c}\left(\rho C_{p}\right)_{h f}=\left(\rho C_{p}\right)_{f}\left(1-\varphi_{2}\right)\left(1-\varphi_{1}\right)+\varphi_{1} \frac{\left(\rho C_{p}\right)_{s 1}}{\left(\rho C_{p}\right)_{f}} \\
+\varphi_{2}\left(\rho C_{p}\right)_{s 2}\end{array}$ \\
\hline $\begin{array}{l}\text { Thermal } \\
\text { conductivity }\end{array}$ & $\frac{k_{n f}}{k_{f}}=\frac{k_{s}+2 k_{f}-2 \varphi\left(k_{f}-k_{s}\right)}{k_{s}+2 k_{f}+\varphi\left(k_{f}-k_{s}\right)}$ & $\begin{array}{l}\frac{k_{h f}}{k_{n 1 f}}=\frac{k_{s 2}+2 k_{n 1 f}-2 \varphi_{2}\left(k_{n 1 f}-k_{s 2}\right)}{k_{s 2}+2 k_{n 1 f}+\varphi_{2}\left(k_{n 1 f}-k_{s 2}\right)} \\
\text { Where } \\
\qquad \frac{k_{n 1 f}}{k_{f}}=\frac{k_{s 1}+2 k_{f}-2 \varphi_{1}\left(k_{f}-k_{s 1}\right)}{k_{s}+2 k_{f}+\varphi_{1}\left(k_{f}-k_{s 1}\right)}\end{array}$ \\
\hline $\begin{array}{l}\text { thermal } \\
\text { expansion } \\
\text { coefficient }\end{array}$ & $\beta_{n f}=\beta_{f}\left((1-\varphi)+\varphi\left(\frac{\beta_{s}}{\beta_{f}}\right)\right)$ & $\beta_{h f}=\beta_{f}\left(1-\varphi_{2}\right)\left(\left(1-\varphi_{1}\right)+\varphi_{1}\left(\frac{\beta_{s 1}}{\beta_{f}}\right)\right)+\varphi_{2} \rho_{s 2}$ \\
\hline
\end{tabular}

Table 2: Thermophysical properties of fluid and nanoparticles [34]

\begin{tabular}{|l|l|l|c|}
\hline Physical properties & Fluid phase(water) & $\mathrm{Cu}$ & $\mathbf{T i O}_{\mathbf{2}}$ \\
\hline $\boldsymbol{C}_{\boldsymbol{p}}(\mathrm{J} / \mathrm{kg} . \mathrm{K})$ & 4179 & 385 & 686.2 \\
\hline $\boldsymbol{\rho}\left(\mathrm{kg} / \boldsymbol{m}^{\mathbf{3}}\right)$ & 997.1 & 8933 & 4250 \\
\hline $\boldsymbol{K}(\mathrm{w} / \mathrm{mk})$ & 0.613 & 400 & 8.9538 \\
\hline $\boldsymbol{\beta} \times \mathbf{1 0}^{-\mathbf{5}}(\mathbf{1} / \boldsymbol{k})$ & 21 & 1.67 & 0.9 \\
\hline
\end{tabular}


The boundary conditions are:

$u=0, \quad v=-v_{0}, \quad N=-s \frac{\partial u}{\partial y} \quad T=T_{1}, \quad C=C_{1} \quad$ at $y=-h$

$u=U(x), \quad N=-s \frac{\partial u}{\partial y} \quad T=T_{2}, \quad C=C_{2} \quad$ at $\quad y \rightarrow h$

When $\mathrm{v}_{0}>0$, suction occurs and when $\mathrm{v}_{0}<0$, injections occur. Here, $\mathrm{s}$ is a boundary parameter and represents the microelements' rotational degree of freedom near the walls. When $s=0$, there is no rotation for microelements which are close to walls.

This situation corresponds to concentrated particle flows. Researchers also have considered other particular cases such as $s=0.5$ and $s=1$, which represent the weak concentration and the turbulent flow, respectively. We introduce these dimensionless parameters:

$\eta=\frac{y}{h}, \quad u=-\frac{v_{0} x}{h} f^{\prime}(\eta), \quad N=\frac{v_{0} x}{h^{2}} g(\eta)$,

$v=v_{o} f(\eta), \quad \theta=\left(T-T_{1}\right) /\left(T_{2}-T_{1}\right), \quad C=\left(C-C_{1}\right) /\left(C_{2}-C_{1}\right)$

where $\mathrm{T}_{2}=\mathrm{T}_{1}+\mathrm{Ax}$ in which $\mathrm{A}$ is constant.

By substituting these expressions into the governing equations and then eliminating the pressure gradient, Eqs. (2)-(5) transform to the coupled system of nonlinear equations:

$$
\begin{aligned}
& \left(\mu_{h f}+k\right) f^{\prime \prime \prime}-\frac{\rho_{h f}}{\rho_{f}}\left(f f^{\prime \prime}-f^{\prime 2}\right)-k \mathrm{~g}^{\prime}-M\left(1-f^{\prime}\right)+G r \theta+G c \phi=0 \\
& \left(\mu_{\mathrm{hf}}+\frac{k}{2}\right) g^{\prime \prime}+\rho_{\mathrm{hf}} \operatorname{Re}\left(f g^{\prime}-f^{\prime} g\right)-\mu_{h f} k\left(f^{\prime \prime}+2 g\right)=0 \\
& \left(\frac{k_{h f}}{k_{f}}+R d\right) \theta^{\prime \prime}+\frac{(\rho C p)_{h f}}{(\rho C p)_{f}} \operatorname{Re} \operatorname{Pr}\left(f \theta^{\prime}-f^{\prime} \theta\right)+\operatorname{PrEc}\left(\mu_{h f}+k\right)\left(M f^{\prime 2}+f^{\prime \prime 2}\right) \\
& +Q \operatorname{Pr} \theta=0 \\
& \left(1-\varphi_{1}\right)\left(1-\varphi_{2}\right) \phi^{\prime \prime}+S c f \phi^{\prime}-\gamma S c \phi=0
\end{aligned}
$$

With boundary conditions

$$
\begin{aligned}
& f(-1)=-1, f^{\prime}(-1)=S, g(-1)=0, \theta(-1)=0, \phi(-1)=0, \\
& f(1)=1, f^{\prime}(1)=0, \quad g(1)=0, \quad \theta(1)=1, \quad \phi(1)=0
\end{aligned}
$$


where $S$ is parameter stretching and shrinking $S<0$ Shrinking case $S>0$ Stretching case, Pr is the Prandtl number, Re is the Reynolds number where Re $>0$ corresponds to suction $\mathrm{n}$ and $\mathrm{Re}<0$ corresponds to injection, $\mathrm{R}$ is the radiation parameter, $\mathrm{M}$ is the magnetic parameter, $\mathrm{K}$ is the micropolar parameter. These parameters are defined as below:

$$
\begin{gathered}
R e=\rho_{f} v_{0} h / \mu_{f}, \quad p r=\frac{\mu_{f} C_{p f}}{k_{f}}, \quad R d=4 \sigma^{*} T_{\infty}^{3} / k_{h f} k_{h f}^{*}, \\
G r=\frac{g B_{f}\left(T_{w}-T_{\infty}\right)}{b u_{w}}, G c=\frac{g B_{f}\left(C_{w}-C_{\infty}\right)}{b u_{w}} \\
M=\sigma_{f} B_{0}^{2} h^{2} / \mu_{f}, \quad K=k / \mu_{f}, \quad j=h^{2}, \quad \gamma=\frac{k_{r}}{b}, \quad E c=\frac{u_{w}^{2}}{c_{\rho}\left(T_{w}-T_{\infty}\right)}, \\
Q=\frac{Q_{0}}{b\left(\rho C_{\rho}\right)_{f}}, \quad S C=\frac{v_{f}}{D_{B}}
\end{gathered}
$$

Above the quantities are inertia coefficient, Gr is Grashof numbers, Rd is Radiation factor, $\mathrm{M}$ is Magnetic factor, $\mathrm{k}$ is the vortex viscosity, $\gamma$ is Chemical reaction factor, $\mathrm{Ec}$ is Eckert number, Pr is Prandtl number, Q is heat source/sink factor, SC is Schmidt number.

Another substantial characteristic of the present work is the skin friction coefficient and Nusselt number as follows:

$$
\begin{aligned}
& C_{f}=\left|\left(\frac{\mu_{h f}}{\mu_{f}}+K\right) f^{\prime \prime}(0)\right| \\
& N u=\left|\frac{k_{h f}}{k_{f}}\left(1+\frac{4}{3} R\right) \theta^{\prime}(0)\right|
\end{aligned}
$$

\section{Result and discussion:}

The governing partial differential equations were transformed into ordinary differential equations. The obtained self-similar equations were solved numerically by using the fourth-order Runge-Kutta-Gill and shooting methods. Results are presented in Tables and Figures to show parametric effects on velocity, angular velocity, temperature, concentration, the skin friction coefficient, and Nusselt number.

From Table 3, we see the skin friction under effective nanoparticle, magnetic parameter, Prandtl number, radiation parameter, rate heat source parameter, and Eckert number in two cases stretching and shrinking. Table 4 also shows the Nusselt number under effective nanoparticle, magnetic parameter, and Grashof number in two cases stretching and shrinking. 
Table 3: effect of $\varphi_{1}, \varphi_{2}, \mathrm{M}$ and $\mathrm{Gr}$ in Skin friction $\mathrm{f}^{\prime \prime}(-1)$

\begin{tabular}{|c|c|c|c|c|c|}
\hline Case & $\varphi_{1}(c u)$ & $\varphi_{2}\left(\mathrm{TiO}_{2}\right)$ & $\mathrm{M}$ & $\mathrm{Gr}$ & $\mathrm{f}^{\prime \prime}(0)$ \\
\hline \multirow{7}{*}{ Stretching } & 0.0 & 0.0 & 0.1 & 0.5 & 0.0989 \\
\hline & 0.05 & 0.05 & & & 0.1510 \\
\hline & 0.1 & 0.1 & & & 0.1733 \\
\hline & 0.1 & 0.1 & 1.0 & & 0.0055 \\
\hline & $"$ & $"$ & 2.0 & & -0.1248 \\
\hline & $"$ & $"$ & & 1.0 & 0.2349 \\
\hline & $"$ & $"$ & & 1.5 & 0.2942 \\
\hline \multirow{7}{*}{ Shrinking } & 0.0 & 0.0 & 0.1 & 0.5 & 0.3576 \\
\hline & 0.05 & 0.05 & & & 0.3262 \\
\hline & 0.1 & 0.1 & & & 0.3045 \\
\hline & $"$ & $"$ & 1.0 & & 0.3842 \\
\hline & $"$ & $"$ & 2.0 & & 0.4560 \\
\hline & $"$ & $"$ & & 1.0 & 0.3362 \\
\hline & $"$ & " & & 1.5 & 0.3683 \\
\hline
\end{tabular}

Table 4: effect of $\varphi_{1}, \varphi_{2}, \mathrm{M}, \mathrm{Rd}, \mathrm{Ec}, \mathrm{Pr}$ and $\mathrm{Q}$ in Nusselt number $\Theta^{\prime}(-1)$

\begin{tabular}{|c|c|c|c|c|c|c|c|}
\hline Case & $\varphi_{1}=\varphi_{2}$ & $\operatorname{Pr}$ & $\mathrm{M}$ & Ec & $\mathrm{Rd}$ & $\mathrm{Q}$ & $\Theta^{\prime}(-1)$ \\
\hline \multirow{13}{*}{ Stretching } & 0.0 & 0.71 & 0.1 & 0.5 & 1.0 & 0.3 & 0.3194 \\
\hline & 0.05 & & & & & & 0.3409 \\
\hline & 0.1 & & & & & & 0.3833 \\
\hline & & 1.0 & & & & & 0.3058 \\
\hline & & 1.5 & & & & & 0.2223 \\
\hline & & & 1.0 & & & & 0.4374 \\
\hline & & & 2.0 & & & & 0.4409 \\
\hline & & & & 1.5 & & & 0.3577 \\
\hline & & & & 2.0 & & & 0.3544 \\
\hline & & & & & 2.0 & & 0.4059 \\
\hline & & & & & 3.0 & & 0.4290 \\
\hline & & & & & & 0.9 & 0.4226 \\
\hline & & & & & & 1.5 & 0.5054 \\
\hline \multirow{13}{*}{ Shrinking } & 0.0 & 0.71 & 0.1 & 0.5 & 1.0 & 0.3 & 0.3444 \\
\hline & 0.05 & & & & & & 0.3652 \\
\hline & 0.1 & & & & & & 0.3833 \\
\hline & & 1.0 & & & & & 0.3457 \\
\hline & & 1.5 & & & & & 0.2829 \\
\hline & & & 1.0 & & & & 0.3937 \\
\hline & & & 2.0 & & & & 0.4021 \\
\hline & & & & 1.5 & & & 0.3787 \\
\hline & & & & 2.0 & & & 0.3741 \\
\hline & & & & & 2.0 & & 0.4228 \\
\hline & & & & & 3.0 & & 0.4412 \\
\hline & & & & & & 0.9 & 0.4475 \\
\hline & & & & & & 1.5 & 0.5210 \\
\hline
\end{tabular}


From Fig. 2, we see the effect of the magnetic parameter $M$ on the velocity in two cases. First, in the stretching case, the magnetic field parameter increases the velocity is decreasing but, in the shrinking case, the magnetic field parameter increases the velocity is increasing, and we see then decreasing is more than increasing. Fig. 3 describes the effect of the hybrid nanoparticles in angular momentum in two cases. In the stretching case, when the volume of hybrid increases, angular velocity decreases but near a boundary surface of the channel, the angular velocity increase, but in the Shrinking case, when the volume of hybrid increases, angular velocity decreases.

Fig. 4 shows the effect of the magnetic parameter $M$ on the angular velocity in two cases. First, in the stretching case, the magnetic parameter increases the angular velocity, but at a certain point, inverse decreases angular velocity with increasing magnetic parameter. In the Shrinking case, the magnetic parameter increases the angular velocity decreases but, at a certain point inverse, increases angular velocity with increasing magnetic parameter. Fig. 5 demonstrates the effect of hybrid nanoparticle concentration on the temperature distribution. As the concentration of nanoparticles increases, the temperature is creasing because of the high thermal conductivity of the nanoparticles. Fig. 6 illustrates the effect of the magnetic parameter on the temperature. As the magnetic parameter increases, the temperature is increasing. From Fig. 7, one may observe the effect of the heat source/sink parameter on the temperature. As the heat source/sink parameter increases, the temperature is increasing. From Fig. 8, it can be seen that see the effect of the Schmidt number parameter on the concentration. As the Schmidt number parameter increases, the concentration is increasing. Finally, Fig. 9 illustrates the influence of chemical reaction parameters on the concentration. As the chemical reaction parameter increases, the concentration is decreasing. 


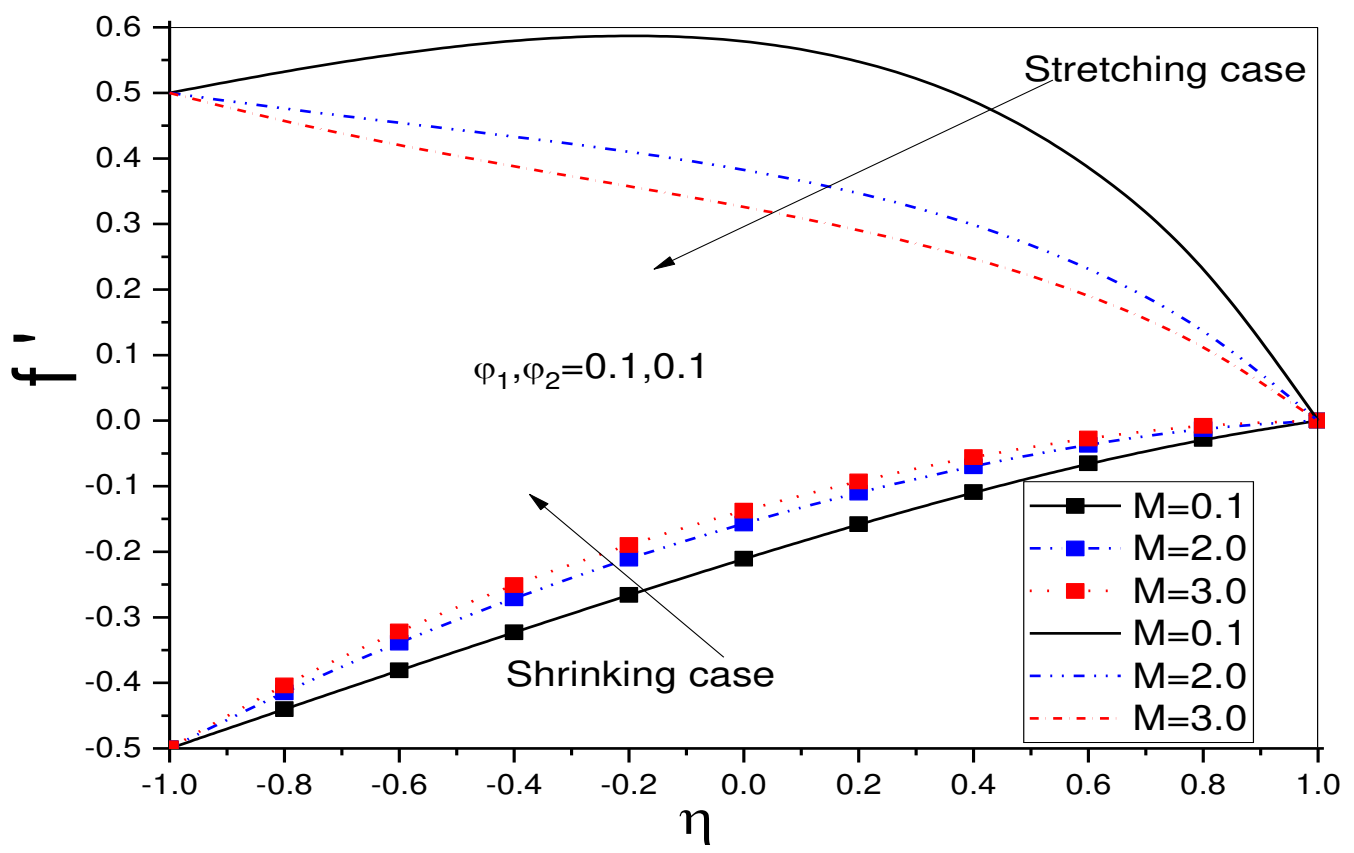

Fig. 2: Effect of the magnetic field parameter on the velocity $\operatorname{Re}=0.1, \operatorname{Pr}=0.71, \mathrm{Q}=0.3$, $\mathrm{Rd}=1.0, \gamma=0.5, \mathrm{Gr}=0.5, \mathrm{Sc}-0.5, \mathrm{Gc}=0.5, \varphi_{1}=0.1, \varphi_{2}=0.1$

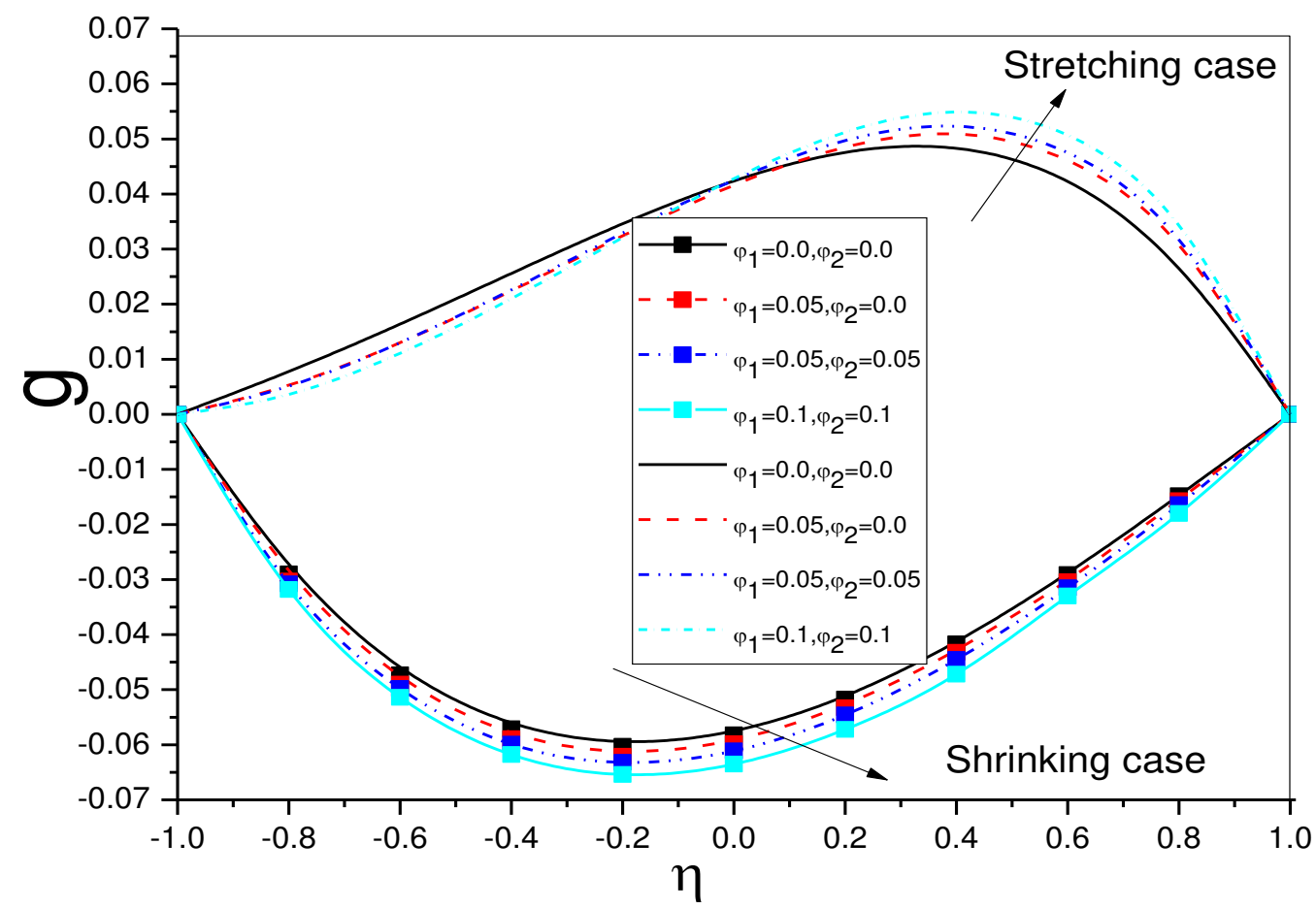

Fig. 3: Effect of nanoparticles on the angular velocity $M=0.1, \operatorname{Re}=0.1, \operatorname{Pr}=0.71, Q=0.3$, $\mathrm{Rd}=1.0, \gamma=0.5, \mathrm{Gr}=0.5, \mathrm{Sc}-0.5, \mathrm{Gc}=0.5$ 


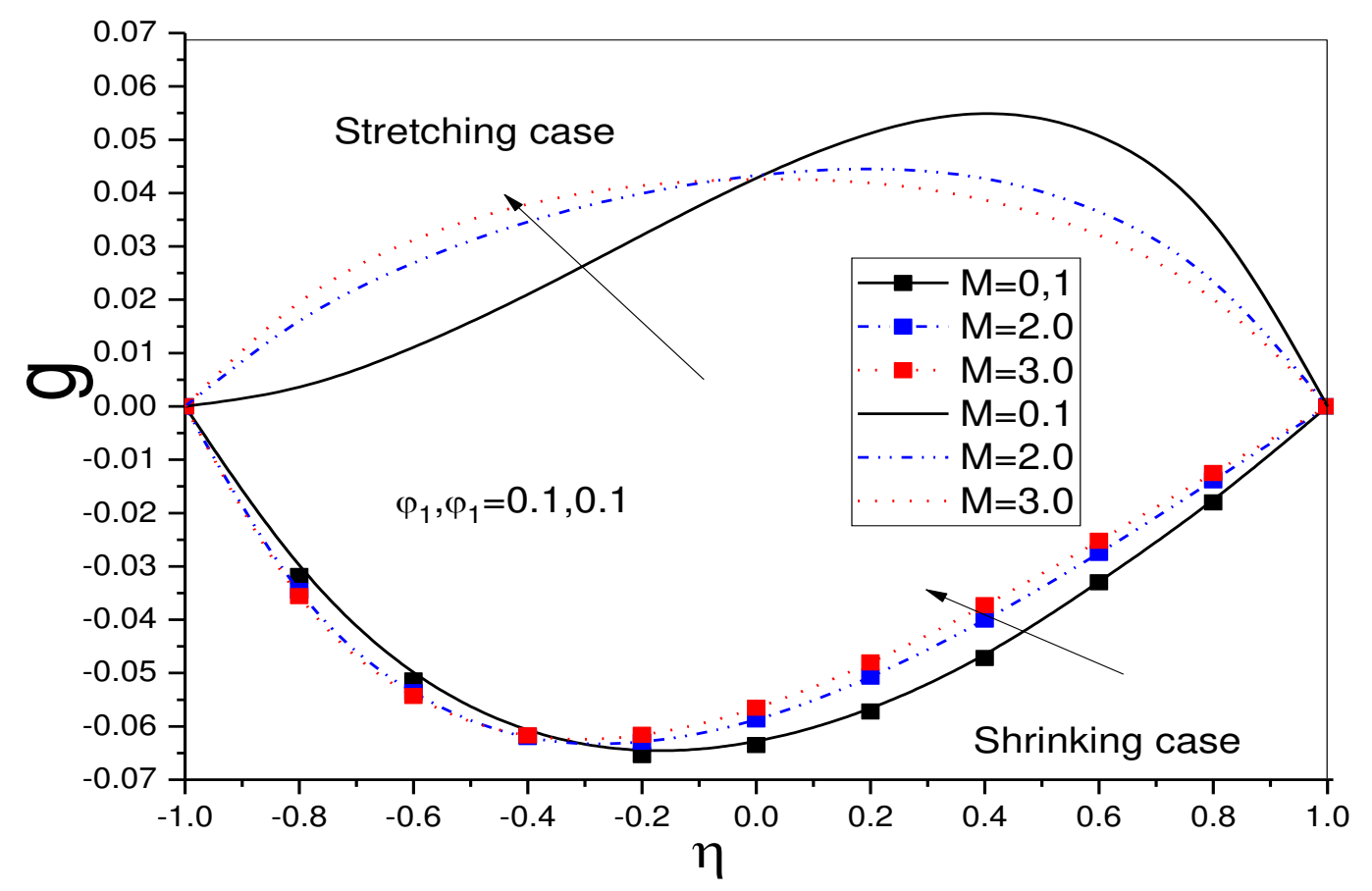

Fig. 4: Effect of the Magnetic field parameter. on the Angular velocity $\mathrm{Re}=0.1, \mathrm{Q}=0.3$, $\operatorname{Pr}=0.71, \mathrm{Rd}=1.0, \gamma=0.5, \mathrm{Gr}=0.5, \mathrm{Sc}-0.5, \mathrm{Gc}=0.5, \varphi_{1}=0.1, \varphi_{2}=0.1$

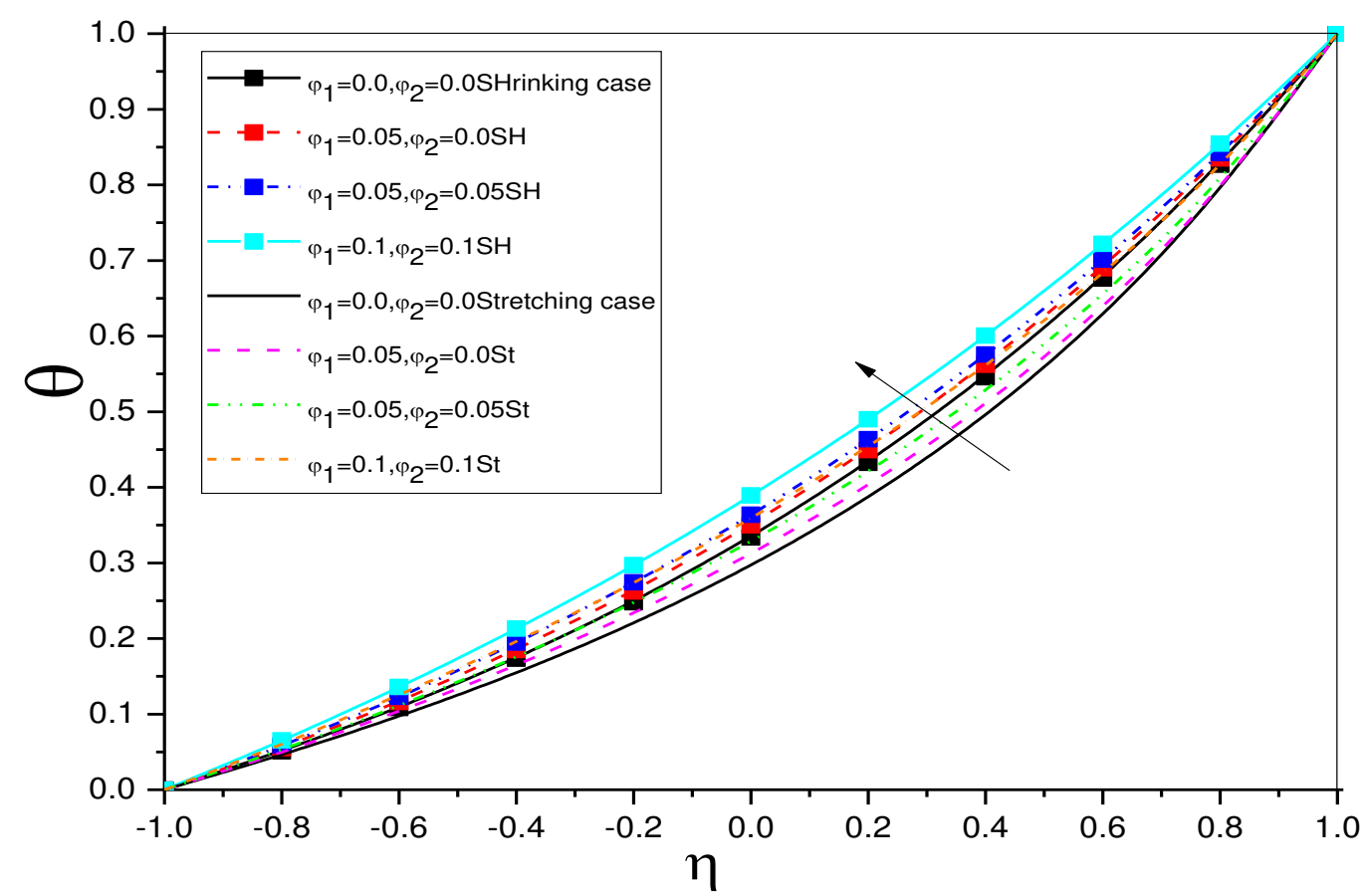

Fig. 5: Effect of nanoparticles on the temperature $M=0.1, \operatorname{Re}=0.1, \operatorname{Pr}=0.71, Q=0.3$, $\mathrm{Rd}=1.0, \gamma=0.5, \mathrm{Gr}=0.5, \mathrm{Sc}-0.5, \mathrm{Gc}=0.5$ 


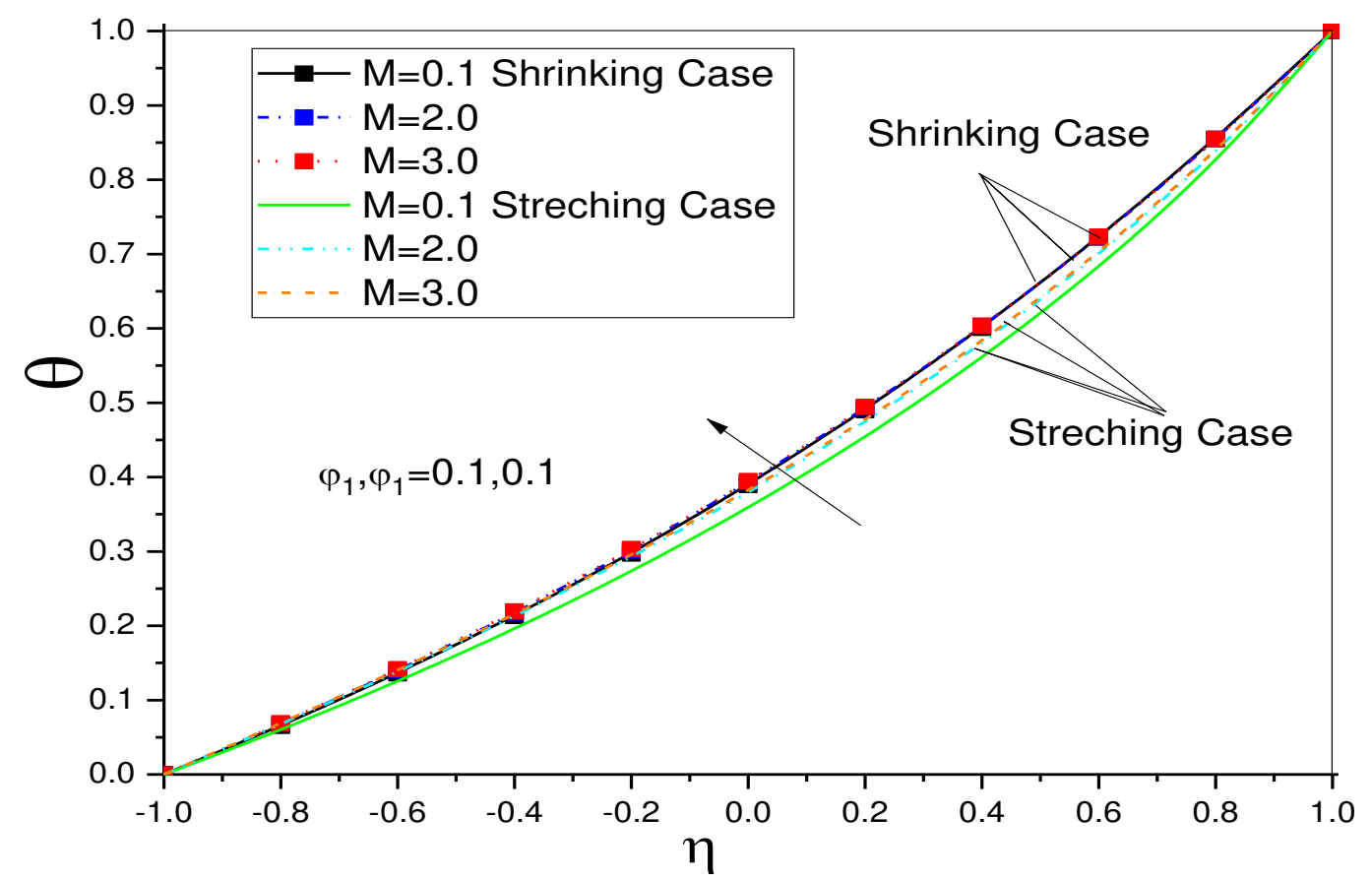

Fig. 6: Effect of the Magnetic field parameter. on the temperature $\operatorname{Re}=0.1, \mathrm{Q}=0.3$, $\operatorname{Pr}=0.71, \mathrm{Rd}=1.0, \gamma=0.5, \mathrm{Gr}=0.5, \mathrm{Sc}-0.5, \mathrm{Gc}=0.5, \varphi_{1}=0.1, \varphi_{2}=0.1$

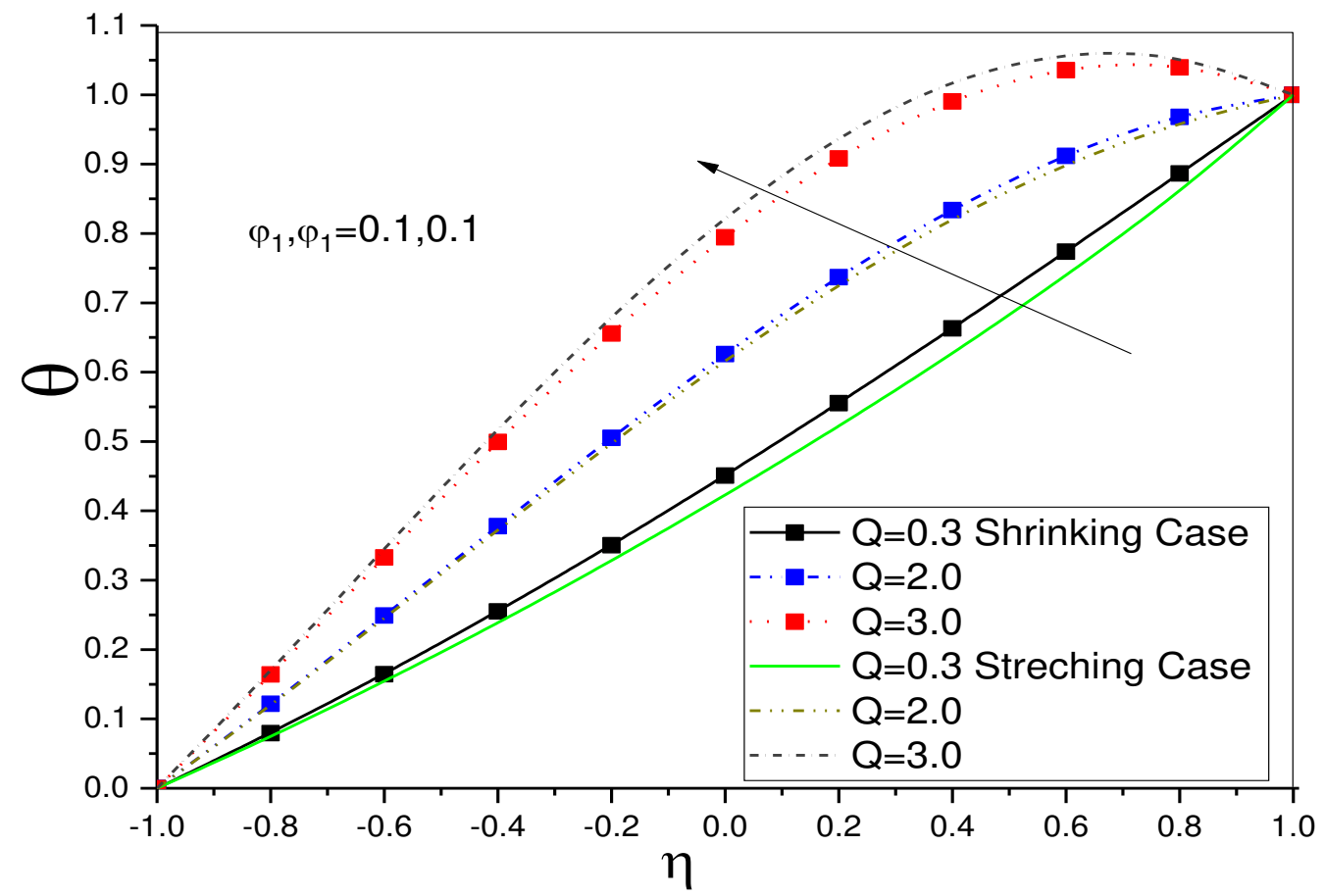

Fig. 7: Effect of the heat source/sink parameter. on the temperature $\operatorname{Re}=0.1, \mathrm{pr}=0.71$, $\mathrm{Rd}=1.0, \gamma=0.5, \mathrm{Gr}=0.5, \mathrm{Sc}-0.5, \mathrm{Gc}=0.5, \varphi_{1}=0.1, \varphi_{2}=0.1$ 


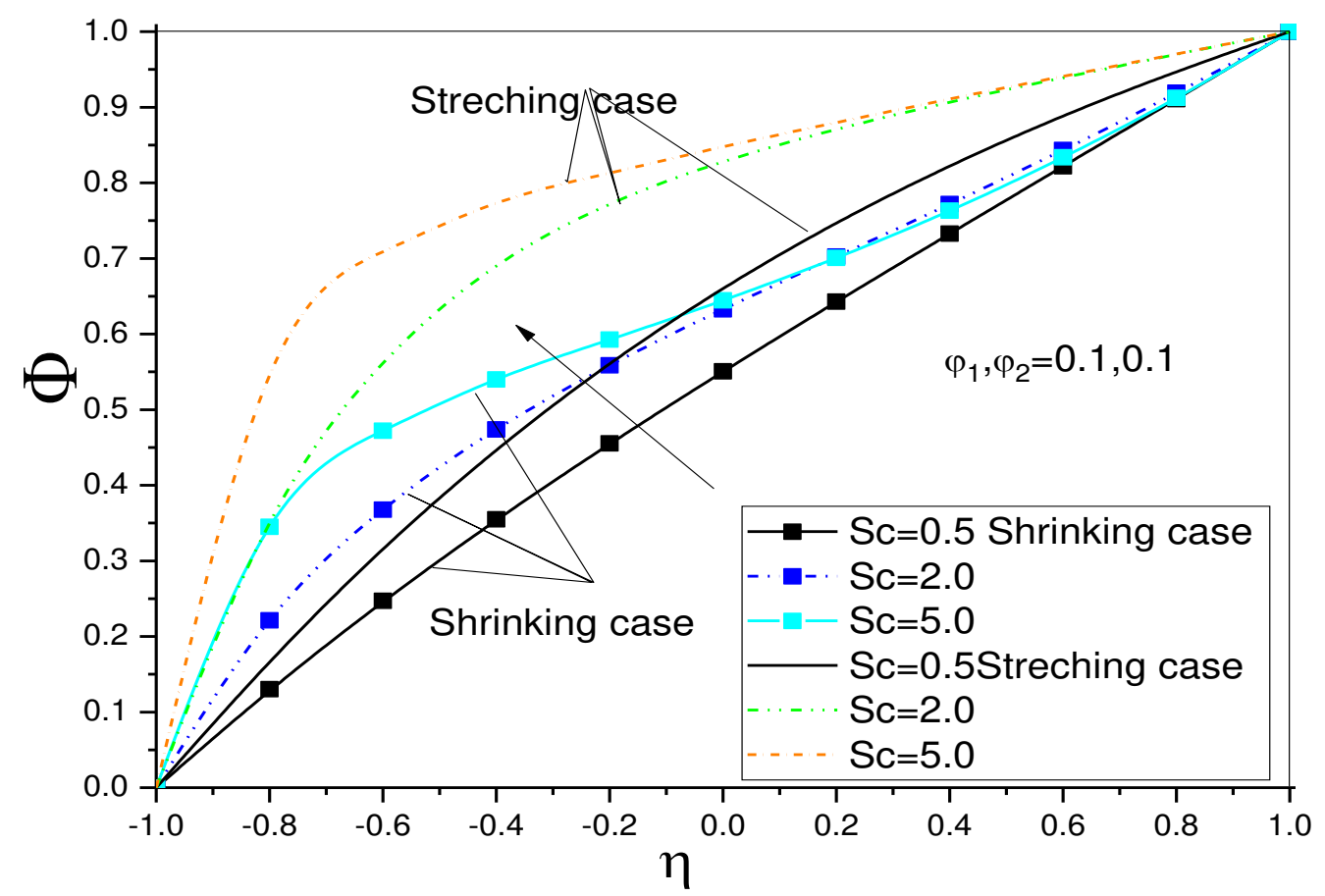

Fig. 8: Effect of the Schmidit number on the temperature $\mathrm{Re}=0.1, \mathrm{Q}=0.3, \mathrm{M}=0.1$, $\operatorname{Pr}=0.71 \gamma=0.5, \mathrm{Gr}=0.5, \mathrm{Rd}-0.5, \mathrm{Gc}=0.5, \varphi_{1}=0.1, \varphi_{2}=0.1$

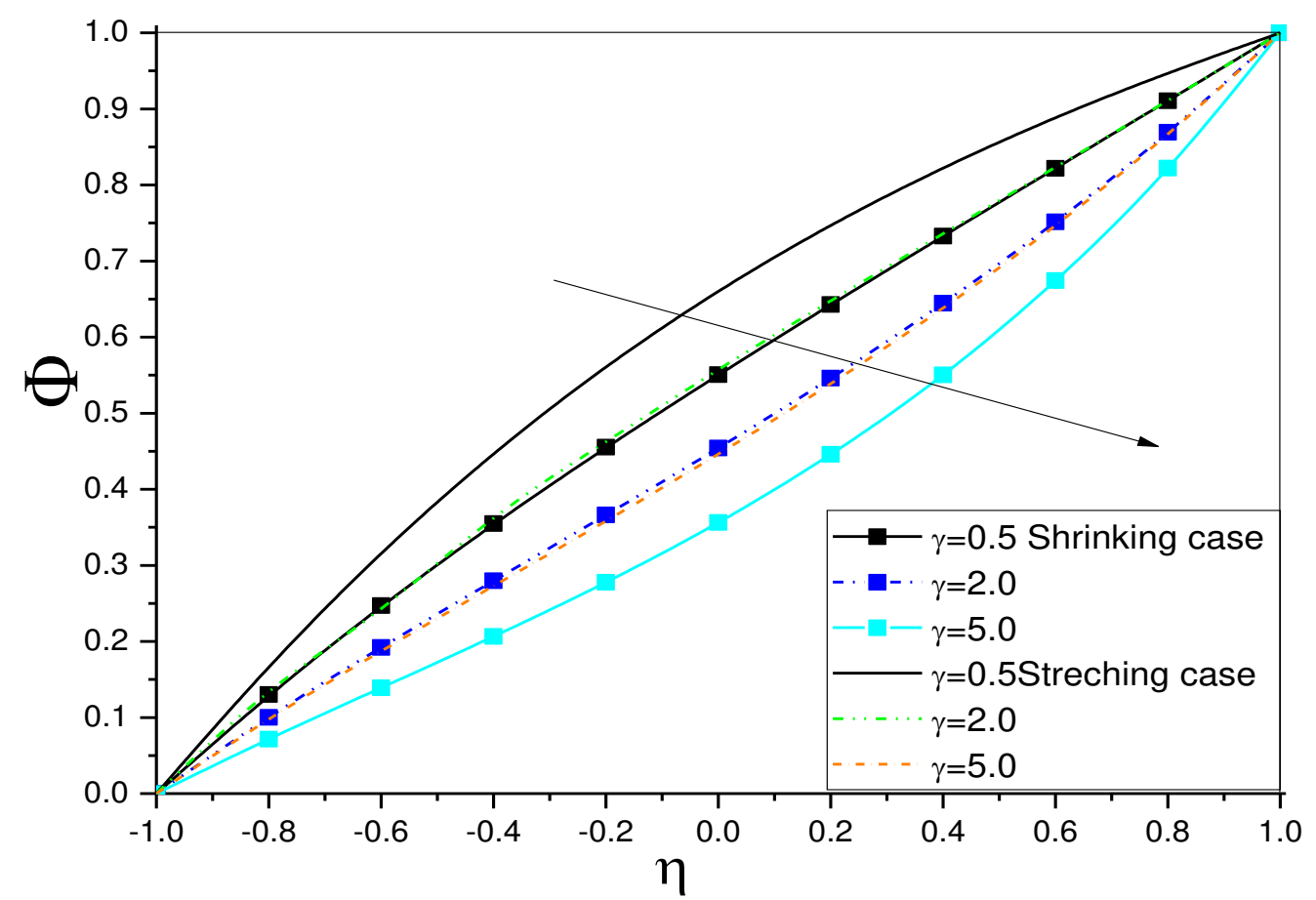

Fig. 9: Effect of the chemical reaction parameter on the temperature $\operatorname{Re}=0.1, \mathrm{Q}=0.3$, $\mathrm{M}=0.1, \mathrm{Pr}=0.71 \mathrm{Sc}=0.5, \mathrm{Gr}=0.5, \mathrm{Rd}-0.5, \mathrm{Gc}=0.5, \varphi_{1}=0.1, \varphi_{2}=0.1$ 


\section{Conclusion:}

In this work, a channel with penetrable walls and radiation contains MHD micropolar hybrid nanofluid flow and heat transfer. This study explores the effects of nanoparticles concentration on velocity, angular velocity, concentration and thermal fields. It was clear that the effect of heat of the hybrid nanofluids is directly proportional to its velocity and angular velocity. We also noticed that not all parameters have an impact on the distribution of the angular momentum.

\section{References:}

[1] C. M. Hussain, Handbook of nanomaterials for industrial applications, Elsevier, USA, 2018

[2] J. Sarkar, P. Ghosh, A. Adil, A review on hybrid nanofluids: Recent research, development and applications, Renewable and Sustainable Energy Reviews, vol. 43, pp. 164-177, 2015.

[3] A.C. Eringen, Theory of micropolar fluids, J. Math. Mech. 16 (1966) 1-18.

[4]G. Łukaszewicz, Micropolar Fluids: Theory and Applications, Springer, Berlin, 1999.

[5] S. Siddiqa, A. Faryad, Naheed Begum, M.A. Hossain, Rama Subba Reddy Gorla, Periodic magnetohydrodynamic natural convection flow of a micropolar fluid with radiation, Int. J. Therm. Sci. 111 (2017) 215-222.

[6] D. Srinivasacharya, K. Hima Bindu, Entropy generation in a porous annulus due to micropolar fluid flow with slip and convective boundary conditions, J. Energy 111 (2016) 165-177.

[7] F. Mabood, S.M. Ibrahim, M.M. Rashidi, M.S. Shadloo, Giulio Lorenzini, Nonuniform heat source/sink and Soret effects on MHD non-Darcian convective flow past a stretching sheet in a micropolar fluid with radiation, Int. J. Heat. Mass Transf. 93 (2016) 674-682.

[8] M. Turkyilmazoglu, Mixed convection flow of magnetohydrodynamic micropolar fluid due to a porous heated/cooled deformable plate: exact solutions, Int. J. Heat. Mass Transf. 106 (2017), pp.127-134.

[9] N. Sandeep, C. Sulochana, Dual solutions for unsteady mixed convection flow of MHD micropolar fluid over a stretching/shrinking sheet with non-uniform heat source/sink, J. Eng. Sci. Technol. 18, (2015) 738-745.

[10] M. Fakour, A. Vahabzadeh, D.D. Ganji, M. Hatami, Analytical study of micropolar fluid flow and heat transfer in a channel with permeable walls, J. Mol. Liq. 204 (2015) 198-204. 
[11] S. Rawat, S. Kapoor, R. Bhargava, MHD flow heat and mass transfer of micropolar fluid over a nonlinear stretching sheet with variable micro inertia density, heat flux and chemical reaction in a non-Darcy porous medium, J. Appl. Fluid Mech. 9 (2016) 321-331.

[12] M. Turkyilmazoglu, Flow of a micropolar fluid due to a porous stretching sheet and heat transfer, Int. J. Non-Linear Mech. 83 (2016) 59-64.

[13]H.S. Takhar, R. Bhargava, R.S. Agrawal, A.V.S. Balaji, Finite element solution of micropolar fluid flow and heat transfer between two porous discs, Int. J. Eng. Sci. 38 (2000) 1907-1922.

[14] R. Ellahi, M. Hassan, A. Zeeshan, A.A. Khan, The shape effects of nanoparticles suspended in HFE- 7100 over wedge with entropy generation and mixed convection, Appl. NanoSci. 6 (2016) 641-651.

[15] M. Akbarzadeh, S. Rashidi, M. Bovand, R. Ellahi, A sensitivity analysis on thermal and pumping power for the flow of nanofluid inside a wavy channel, J. Mol. Liq. 220 (2016) 1-3.

[16] R. Ellahi, M. Hassan, A. Zeeshan, Aggregation effects on water base Al2O3nanofluid over permeable wedge in mixed convection, Asia Pac. J. Chem. Eng. 11 (2016) 179-186.

[17] Mohsen Sheikholeslami, Davood Domiri Ganji, Nanofluid flow and heat transfer between parallel Plates considering Brownian motion using DTM, Comput. Methods Appl. Mech. Eng. 283 (2015) 651- 663.

[18] M. Sheikholeslami, D.D. Ganji, Nanofluid hydrothermal behavior in existence of Lorentz forces considering Joule heating effect, J. Mol. Liq. 224 (2016) 526-537. [19] A. Rahbari, M. Fakour, A. Hamzehnezhad, M. Akbari Vakilabadi, D.D. Ganji, Heat transfer and fluid flow of blood with nanoparticles through porous vessels in a M. Alizadeh et al. Case Studies in Thermal Engineering 12 (2018) 319-332.

[20] M.M. Bhatti, A. Zeeshan, R. Ellahi, EndoScope analysis on peristaltic blood flow of Sisko fluid with Titanium magneto-nanoparticles, Comput. Biol. Med 78 (2016) $29-41$.

[21] M.M. Bhatti, A. Zeeshan, R. Ellahi, Simultaneous effects of coagulation and variable magnetic field On peristaltically induced motion of Jeffrey nanofluidcontaining gyrotactic microorganism, MicrovaSc. Res. 110 (2017) 32-42. [22] M.M. Bhatti, S.R. Mishra, T. Abbas, M.M. Rashidi, A mathematical model of MHD nanofluid flow having gyrotactic microorganisms with thermal radiation and chemical reaction effects, Neural Compute. Appl. (2016), 
http://dx.doi.org/10.1007/s00521-016-2768-8.

[23] M.M. Bhatti, M.M. Rashidi, Effects of thermo-diffusion and thermal radiation on Williamson nanofluid over a porous shrinking/stretching sheet, J. Mol. Liq. 221 (2016) 567-573.

[24] M.M. Rashidi, N.V. Ganesh, A.A. Hakeem, B. Ganga, Buoyancy effect on MHD flow of nanofluid over a stretching sheet in the presence of thermal radiation, J. Mol. Liq. 198 (2014) 234-238.

[25] M.A. Sheremet, I. Pop, N.C. Roşca, Magnetic field effect on the unsteady natural convection in a wavy-walled cavity filled with a nanofluid: buongiorno's mathematical model, J. Taiwan Inst. Chem. Eng. 61 (2016) 211-222.

[26] M.A. Sheremet, T. Grosan, I. Pop, Free convection in a square cavity filled with a porous medium saturated by nanofluid using Tiwari and Das' nanofluid model, Transp. Porous Med. 106 (2015) 595-610.

[27] M.A. Sheremet, I. Pop, M.M. Rahman, Three-dimensional natural convection in a porous enclosure filled with a nanofluid using Buongiorno's mathematical model, Int. J. Heat. Mass Transf. 82 (2015) 396-405.

[28] A.S. Dogonchi, D.D. Ganji, Analytical solution and heat transfer of two-phase nanofluid flow between Non-parallel walls considering Joule heating effect, Powder Technol. 318 (2017) 390-400.

[29] A.S. Dogonchi, M. Alizadeh, D.D. Ganji, Investigation of MHD Go-water nanofluid flow and heat transfer in a porous channel in the presence of thermal radiation effect, Adv. Powder Technol. 28 (2017) 1815- 1825.

[30] A.S. Dogonchi, D.D. Ganji, Study of nanofluid flow and heat transfer between non-parallel stretching walls considering Brownian motion, J. Taiwan Inst. Chem. Eng. 69 (2016) 1-3.

[31]H.A. El-dawy. et. al Stagnation Point Thermal Boundary Layer Flow Towards a Stretching/Shrinking Sheet in a Nanofluid Journal of Nanofluids Vol. 2, pp. 1-5, 2013

[32]H.A. El-dawy, R.gorla, Unsteady Flow of a Nanofluid Over ahrinking/Stretching Porous Wedge Sheet in the Presence of Solar Radiation Journal of Nanofluids Vol . 7, pp. 1-9, 2018

[33] Ahmdi, et al. "Configuration and optimization of a Minichannel using wateralumina nanofluid by non-dominated sorting genetic algorithm and response surface method." Nanomaterials 10, no. 5 (2020): 901

[34]H. Maleki, et al. "Flow and heat transfer in non-Newtonian nanofluids over 
porous surfaces." Journal of Thermal Analysis and Calorimetry 135, no. 3 (2019): 1655-1666.

[35] Marjan Goodarzi, et el "Efficiency assessment of using graphene nanoplateletssilver/water nanofluids in microchannel heat sinks with different cross-sections for electronics cooling." International Journal of Numerical Methods for Heat \& Fluid Flow (2019). 